\title{
COMPLETE MODELS OF DIFFERENTIAL FIELDS $\left({ }^{1}\right)$
}

\author{
BY \\ PETER BLUM
}

Introduction. This work constitutes a step in the direction of a "global" theory of differential algebra. The main result (Theorem 1 of \$2) is the following: Let $K$ be an ordinary differential field of characteristic zero. Then there is a premodel of $K$ with the property that every maximal differential homomorphism from a differential subring of $K$, whose differential field of quotients is $K$, induces a point on this pre-model. Under a certain condition these points will be uniquely determined.

It is not yet known whether there exist differential fields of the form $k\left\langle x_{1}, \ldots, x_{n}\right\rangle$, where the $x_{\imath}$ belong to some universal differential field extension of $k$, which do not admit complete models over $k$. Even in the case where $x_{1}, \ldots, x_{n}$ are differentially algebraically independent the answer is not apparent. For definitions, see the discussion preceding the above-mentioned Theorem 1.

The author would like to thank Professors A. Seidenberg and E. R. Kolchin for their helpful comments and suggestions.

Notation AND Conventions. We denote the integers, rationals, reals, and complex numbers by $Z, Q, R, C$, respectively, and usually consider $Z$ as a differential ring, and $Q, R$, and $C$ as differential fields of constants. If $R$ is an integral domain, we write "qf $(R)$ " for its quotient field. If $S$ is a differential integral domain, " $S\{y\}$ " will denote the ring of differential polynomials with coefficients in $S$. The letter " $y$ " will represent a differential indeterminate, while " $x$ ", " $z$ " will stand for differential quantities which may or may not be differential indeterminates.

We begin by examining some of the elementary properties of the category $D$ of differential rings and differential homomorphisms (these are ordinary differential rings, although many of the arguments remain valid in the partial case). For the moment, no restriction is placed on zero divisors or characteristic.

First of all, $D$ admits coproducts in the sense that if $A, B \in D$ we can define $A \otimes B$ by taking the usual tensor product of $A$ and $B$ (considered as $Z$-algebras) and setting $(a \otimes b)^{\prime}=a^{\prime} \otimes b+a \otimes b^{\prime}$. This makes $A \otimes B$ into a differential ring satisfying the conditions for a coproduct in a category. In fact, we have differential

Received by the editors December 8, 1967 and, in revised form, March 7, 1967.

(1) Research partially supported by Office of Naval Research Contract 222 (62), and National Science Foundation Grants GP-3990, GP-3982, and GP-6535. 
homomorphisms $A, B \rightarrow A \otimes B$ by $a \mapsto a \otimes 1, b \mapsto 1 \otimes b$ and the universal property

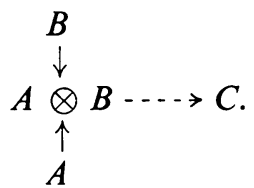

Note that it is not obvious that every ring in $D$ has a differential prime since a maximal differential ideal need not be prime as the following example shows: let $k$ be a differential field of characteristic $p>0$, and in $k\{y\}$ consider the differential ideal $I=\left[y^{p}, y^{\prime}-1\right]$. It is clear that $1 \notin I$, and hence (by Zorn's lemma) $I$ is contained in a maximal differential ideal $M$; but $y^{\prime}-1 \in M$ and hence $y \notin M$; since $y^{p} \in M$, $M$ cannot be prime.

Of course, this cannot happen in characteristic zero: if $M$ is a maximal differential ideal of a differential overring of $Q$, then $M$ is prime. To see this, first observe that $M$ is perfect (because in this case the perfect differential ideal generated by $M$ does not contain 1 and hence coincides with $M$ ), and then recall that every perfect differential ideal is an intersection of prime differential ideals.

In general, a differential ring $A$ contains a prime differential ideal provided $A$ has no nilpotent elements. Indeed, then $(0)$ is a perfect differential ideal and hence is an intersection of prime differential ideals.

Although a maximal differential ideal $M$ need not be prime, we can at least prove that $M$ is primary: suppose $x z \in M, x \notin M$; then $[M, x]=(1)$ so that

$$
(+): 1=m+r_{0} x+r_{1} x^{\prime}+\cdots+r_{n} x^{(n)} \text {. }
$$

But by Raudenbush's lemma (see $\left[2\right.$, p. 8, §10]) $z^{k+1} x^{(k)} \in M$ for all $k$. Hence from $(+)$ we get $z^{n+1}=m z^{n+1}+r_{0} x z^{n+1}+\cdots+r_{n} x^{(n)} z^{n+1} \in M$ which finishes the proof.

It follows that even the weaker statement that every maximal differential ideal is perfect, is false. For a differential ideal which is primary and perfect is prime.

Next we show the existence of inductive limits in $D$. If $\left\{A_{\alpha}, \phi_{\alpha \beta}\right\}$ is a filtering inductive system of differential rings and differential homomorphisms, let $A$ $=$ ind $\lim A_{\alpha}$ in the category of rings and for each $\alpha$, let $\phi_{\alpha}: A_{\alpha} \rightarrow A$ denote the canonical ring homomorphism. If $\phi_{\lambda}\left(a_{\lambda}\right)=\phi_{\mu}\left(a_{\mu}\right)$ there is a $\nu$ such that $\phi_{\lambda \nu}\left(a_{\lambda}\right)$ $=\phi_{\mu v}\left(a_{\mu}\right)$, so that

$$
\phi_{\lambda}\left(a_{\lambda}^{\prime}\right)=\phi_{v}\left(\phi_{\lambda v}\left(a_{\lambda}^{\prime}\right)\right)=\phi_{v}\left(\phi_{\lambda v}\left(a_{\lambda}\right)^{\prime}\right)=\phi_{v}\left(\phi_{\mu v}\left(a_{\mu}\right)^{\prime}\right)=\phi_{v}\left(\phi_{\mu v}\left(a_{\mu}^{\prime}\right)\right)=\phi_{\mu}\left(a_{\mu}^{\prime}\right) .
$$

Therefore, if $a \in A$ we may define $a^{\prime}$ by choosing any $\lambda$ and any $a_{\lambda} \in A_{\lambda}$ with $a=\phi_{\lambda}\left(a_{\lambda}\right)$ and then setting $a^{\prime}=\phi_{\lambda}\left(a_{\lambda}^{\prime}\right)$. If $a, b \in A$ then we can write $a=\phi_{\lambda}\left(a_{\lambda}\right)$, $b=\phi_{\lambda}\left(b_{\lambda}\right)$ for a suitable $\lambda$, so that $a b=\phi_{\lambda}\left(a_{\lambda} b_{\lambda}\right)$, whence $(a b)^{\prime}=\phi_{\lambda}\left(a_{\lambda}^{\prime} b_{\lambda}+a_{\lambda} b_{\lambda}^{\prime}\right)$ $=a^{\prime} b+b^{\prime} a$. Thus, $A$ is now a differential ring and each $\phi_{\alpha}$ is a differential homomorphism. This establishes the existence of inductive limits in $D$.

Next we show that localization is possible in $D$ without restriction on zero divisors. If $A$ is any differential ring and $S$ is a multiplicative system in $A$, then in 
$S^{-1} A$ we define $(a / s)^{\prime}=\left(s a^{\prime}-a s^{\prime}\right) / s^{2}$. To see that this makes sense, suppose $a / s=b / t$ so that there exists an $r \in S$ such that $r(a t-b s)=0$. First check that $(a / s)^{\prime}=\left(a s_{1} / s s_{1}\right)^{\prime}$. Then one has $(a / s)^{\prime}=(r a t / r s t)^{\prime}=(r b s / r s t)^{\prime}=(b / t)^{\prime}$. This completes the proof.

1. Differential places. In this section we study the relationship between derivations and integral closure by pointing out some of the difficulties involved and proposing a definition of "differentially integral" for elements of the quotient field of a differential integral domain. We assume throughout this section that every differential ring under consideration contains $Q$.

One reason for not defining "differentially integral" to mean "integral" is that Seidenberg [3, p. 172] has shown that if $R$ is a differential domain with differential field of quotients $K$, then the integral closure $R^{\prime}$ of $R$ need not be closed under the derivation on $K$.

A second problem concerns morphisms. We know that integral closures are intimately related to valuation rings which, in turn, are tied up with ring homomorphisms. But the morphisms in $D$ must commute with the derivation and this leads us to make the following definition: let $R$ and $K$ be as above, $\phi: R \rightarrow \phi(R)$ a differential homomorphism whose image is also a differential domain. $\phi$ is called a differential place of $K$ if $\phi$ cannot be properly extended to a differential homomorphism of a larger differential subring of $K$ into an extension of the differential quotient field of $\phi(R)$. The domain of $\phi$ (denoted " $R_{\phi}$ ") is called the ring of the differential place $\phi$.

By Zorn's lemma, every differential homomorphism from a differential domain $R$ can be extended to a differential place of $\mathrm{qf}(R)$.

If $S$ is a differential domain containing $Q$, we define the differential integral closure of $S$ (in its differential field of quotients $K$ ) to be the intersection of all the rings of differential places of $K$ which contain $S$, and we denote this intersection by $S^{\prime}$. Now to characterize the elements of $S^{\prime}$.

First, let $\phi: R \rightarrow K^{\prime}$ be a differential place of $K$. Then $R$ is a local differential ring since $\operatorname{Ker} \phi$ is prime and $\phi$ can be extended to $R_{\operatorname{Ker}_{\phi}}$ by $\phi(a / s)=\phi(a) / \phi(s)$ which is again a differential homomorphism. Thus $R$ is local and $\operatorname{Ker} \phi$ is its maximal ideal, so that the image $F=\phi(R)$ is a differential field.

If $a \in K-R$, we have the diagram

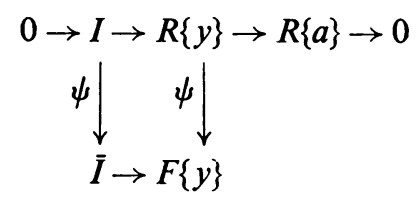

where $I$ is the prime differential ideal of relations satisfied by $a$ over $R ; \psi$ is the differential homomorphism obtained by applying $\phi$ to the coefficients of an element of $R\{y\}$. $I \neq(0)$, or else we could extend $\phi$ to $R\{a\}$. Thus $\bar{I}=\psi(I)$ is a differential ideal in $F\{y\}$ (since $\psi$ is surjective) and the top row is exact. If $1 \notin \bar{I}, \bar{I}$ has a 
zero $x$ in an extension of $F$, and we can complete the bottom line of the above diagram to the bottom line of the following diagram.

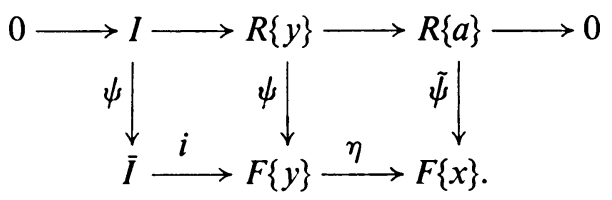

Now $\eta \circ i=0$ so we can define $\tilde{\psi}$ by $\tilde{\psi}(f(a))=(\eta \psi)(f(y))$. Then $\tilde{\psi}$ is a differential homomorphism which properly extends $\phi$. This contradiction shows that $\bar{I}=(1)$, i.e. there exists a differential polynomial $f \in I$ such that $\psi(f)=1$. Let us agree to call a differential polynomial $f \in K\{y\} \phi$-special if $f \in R\{y\}$ and $\psi(f)=1$. Then we have shown that if $a \in K-R$, then $a$ is a zero of a $\phi$-special polynomial.

Conversely, if $a \in K$ is a zero of a $\phi$-special differential polynomial $f$ then $a \notin R$, for otherwise we should have $0=\phi(f(a))=\psi(f)(\phi(a))=1$.

Hence we can state

THEOREM 1. If $S$ is a differential integral domain containing the rationals, with quotient field $K$, then $a \in K$ is differentially integral over $S$ if and only if for every differential place $\phi$ of $K$ finite on $S$ and every $\phi$-special differential polynomial $f$, $f(a) \neq 0$.

Thus we have described the elements of $S^{\prime}$.

Note that if the derivation is trivial we have shown that $a$ is integral over $S$ if and only if $a$ does not satisfy any $\phi$-special polynomial where $\phi$ is a place of $K$ finite on $S$. Monic polynomials of positive degree are never $\phi$-special.

Since every differential homomorphism can be extended to an ordinary place of $K$, we see that every ring of a differential place is contained in a valuation ring.

If $R \subset S \subset \mathrm{qf}(R)$ are differential rings (it is understood that the derivation on $R$ is the restriction of that on $S$ ), then $S$ is differentially integral over $R$ if every element of $S$ is. That is, for all $a \in S$, if $\phi$ is a differential place of qf $(R)$ finite on $R$, then $\phi$ is finite on $a$. We write $\phi(a)=\infty$ to indicate $a \notin R_{\phi}$.

REMARK. Let $S$ be differentially integral over $R, M$ a multiplicative system in $R$, then $M^{-1} S$ is differentially integral over $M^{-1} R$.

Proof. If $x / m \in M^{-1} S$ and $\phi$ is a differential place of qf $(R)$ finite on $M^{-1} R$, then $R \subset M^{-1} R$ implies $\phi(x) \neq \infty$ and $\phi(m) \neq \infty, 0$ (if $\phi(m)=0, m$ is a nonunit in $R_{\phi}$ so that $\phi(1 / m)=\infty$; but $\left.1 / m \in \dot{M}^{-1} R\right)$. Therefore $\phi(x / m) \neq \infty$. Q.E.D.

Let $S$ be differentially integral over $R$ and let $p \subset R$ be a differential prime ideal. Then the differential homomorphism $R \rightarrow R / p$ extends to a differential place $\phi$ of the quotient field of $R$, and by hypothesis $S \subset R_{\phi}$. Let $M$ be the maximal ideal in $R_{\phi}$ and set $P=M \cap S$. Then $P$ is a prime differential ideal in $S$ lying over $p$. Thus the Lying Over Theorem is true for differential primes.

Let $k$ be an algebraically closed field of characteristic zero and make $k[X]$ into a differential ring by setting $X^{\prime}=f(X)$ where $f(X)$ is an arbitrary element of $k[X]$, 
and $c^{\prime}=0, c \in k$. Recall that every local ring between $k[X]$ and $k(X)$ is a discrete valuation ring. It follows that every differential place of $k(X)$ finite on $k[X]$ is a differential homomorphism which is also a place. Conversely, any differential homomorphism which is a place of $k(X)$ (or any other differential field) is a differential place.

Proposition 1. $k[X]$ with this differential structure is differentially integrally closed if and only if $X^{\prime}=0$.

Proof. There is a one-to-one correspondence between nontrivial differential places finite on $k[X]$ and roots of $X^{\prime}$ given by taking the place at each root. If $X^{\prime} \neq 0$, then there are a finite number of roots (if any) of $X^{\prime}$, say $r_{1}, r_{2}, \ldots, r_{n}$ with corresponding differential places $\phi_{1}, \phi_{2}, \ldots, \phi_{n}$. Then $k[X]^{\prime}=\bigcap_{i=1}^{n} R_{\phi_{1}}>\bigcap_{\phi} R_{\phi}$ $=k[X]$, the last intersection being taken over all places $\phi$ of $k(X)$ finite on $X$. If $X^{\prime}=0$, then for all $c \in k, M_{c}=\{g(X) / h(X) \mid h(c) \neq 0, g(c)=0\}$ is a differential ideal in the ring of the place at $c$, so that every place is differential. Q.E.D.

Proposition 2. (a) If $A$ is a differential integral domain, then $\left(A^{\prime}\right)^{\prime}=A^{\prime}$.

(b) Let $A, B, C$ be differential integral domains such that $C \subset B \subset A \subset \mathrm{qf}(C)$. If $A$ is differentially integral over $B$ and $B$ is differentially integral over $C$, then $A$ is differentially integral over $C$.

(c) If $B \subset A \subset \mathrm{qf}(B)$ are differential integral domains, then $B^{\prime} \subset A^{\prime}$.

Proof. (a) Any differential place finite on $A^{\prime}$ is finite on $A$ and conversely. (b) and (c), clear.

Proposition 3. Let $P_{1}^{\prime}, P_{2}^{\prime}$ be distinct differential primes in $A^{\prime}$ both lying over $p \subset A$. Then $A_{p}$ is not differentially integrally closed.

Proof. We know that $A_{P_{i}}^{\prime}$ dominates $A_{p}, i=1,2$ (i.e., they contain $A_{p}$ and $\left.P_{i}^{\prime} A_{P_{i}}^{\prime} \cap A_{p}=p A_{p}\right)$. If $S=A-p, S^{-1} A^{\prime}$ is differentially integral over $A_{p}$. If $A_{p}$ were differentially integrally closed, we should have $S^{-1} A^{\prime}=A_{p}$, but if $r \in P_{1}^{\prime}-P_{2}^{\prime}$, then $r / 1 \in S^{-1} A^{\prime}-A_{p}$. Contradiction.

If $\phi: R_{\phi} \rightarrow K^{\prime}$ is a differential place, let $S \subset R_{\phi}\{y\}=A$ be the set of $\phi$-special polynomials in $y$. Then $S$ is a multiplicative system in $A$ and $S^{-1} A$ has a natural structure of differential ring. $a \in \mathrm{qf}\left(R_{\phi}\right)$ is not in $R_{\phi}$ if and only if $a$ is a zero of an element of $A$ that is a unit in $S^{-1} A$. The differential homomorphism $\tilde{\phi}: A \rightarrow K^{\prime}\{y\}$ induced by $\phi$ maps each element of $S$ onto 1 ; hence $\bar{\phi}$ extends to a differential homomorphism $j: S^{-1} A \rightarrow K^{\prime}\{y\}$.

Let $S \supset R$ be differential rings. We say $S$ covers $R$ if over every prime differential ideal in $R$ there lies a prime differential ideal in $S$. Then $a$ is differentially integral over $R$ if and only if $R\{a\}$ covers $R$ and $a$ satisfies the condition: if $a$ is in the ring of a differential place $\phi$ finite on $R$, then $a$ is in all rings of differential places extending $\left.\phi\right|_{R}$.

EXAMPLE. Let $k$ be a differential field of characteristic zero (ordinary or partial; if it is partial then "'" " denotes any of the derivations). Let $x$ be a differential 
quantity in an extension field of $k$ and set $R=k\{x\}$. Then it is a special case of a result of Seidenberg (Algebraic proof of a theorem of Ritt, unpublished) that $R\left\{x^{\prime} / x\right\}$ covers $R$.

Of course, it is not true that any homomorphism from $R$ into a differential field can be extended to $R\left\{x^{\prime} / x\right\}$. In fact, if $R$ is any unique factorization domain and $a / b \in \mathrm{qf}(R)-R$ is in lowest terms, let $p$ be an irreducible factor of $b$. Then $R$ $\rightarrow R /(p)$ cannot be extended to $a / b$.

One might conjecture that if $R\{x\}$ covers $R$, then $x$ is differentially integral over $R$. The following example shows that this and its algebraic analogue (which amounts to the converse of the Lying Over Theorem) are false: take any singular curve whose normalization has two points over the singular point

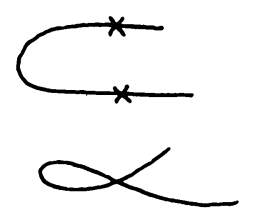

Let $R$ be the local ring of the singularity and $S$ its integral closure. Suppose $\widetilde{P} \neq \widetilde{Q}$ both lie over $P \subset R$. Choose $t \in \widetilde{P}-\widetilde{Q}$. Then $1 / t$ is not integral over $R$, or else we would have $1 / t \in S$ and then $1=t(1 / t) \in \widetilde{P}$. We show that over every prime ideal $P$ of $R$ there lies a prime ideal in $R[1 / t]$.

Proof. Let $M=\left\{t, t^{2}, \ldots, t^{n}, \ldots\right\}$ and consider the diagram

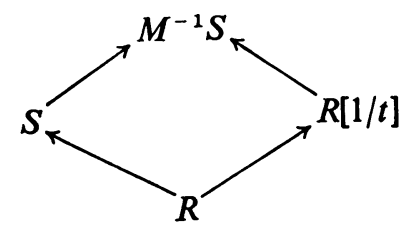

$P$ is the only prime in $R$. Since $\widetilde{Q} \cap M=\varnothing$, there exists a prime $\widetilde{Q}^{\prime}$ in $M^{-1} S$ lying over $\tilde{Q}$. Then $\tilde{Q}^{\prime} \cap R[1 / t] \cap R=P$. Q.E.D.

Note that this also provides a counterexample to the differential algebraic question, for if we take the trivial derivation, "integral closure" equals "differential integral closure" and primes are the same as differential primes.

Now we turn to the relationship between localizing and passing to the differential integral closure.

If $S$ is a multiplicative system in a differential domain $R$, then since $S^{-1} R^{\prime}$ is differentially integral over $S^{-1} R$ we have $S^{-1} R^{\prime} \subset\left(S^{-1} R\right)^{\prime}$. Let $C\left(R^{\prime}, R\right)$ be the conductor of $R$ in $R^{\prime}$. $C=\left\{r \in R \mid r R^{\prime} \subset R\right\}=C\left(R^{\prime}, R\right)$ is a differential ideal in $R$ and $R^{\prime}$. If $s \in C \cap S$, then $R^{\prime} \subset(1 / s) R \subset S^{-1} R$. Hence, if $C \cap S \neq \varnothing$, then $S^{-1} R^{\prime}=S^{-1} R$. 
2. Complete models of differential fields. Let $K$ be an ordinary differential field of characteristic zero and let $k$ be a differential subring of $K$. The affine model determined by the ring $k\{y\}$, that is, the set of all $k\{y\}_{\mathscr{L}}$ as $\mathscr{L}$ ranges over the prime differential ideals in $k\{y\}$, is not complete in the sense that there exist differential places of the quotient field which have no center on $k\{y\}$. For example, any differential place sending $1 / y$ to zero. We begin the study of this situation with the observation that certain algebraic facts can be carried over to the differential case.

Let $A \subset B$ be local differential rings. By this we mean that their maximal ideals consist of all the nonunits and are differential ideals, but this latter assumption will not be needed for the moment. Let $m(A), m(B)$ denote their maximal ideals. Then the following are equivalent: (1) $m(B) \cap A=m(A),(2) m(A) \subset m(B),(3) 1 \notin m(A) \cdot B$. If any of these holds, we say $B$ dominates $A$ (written $B \geqq A$ ).

If $K$ is a differential field as above and $A \subset K$ is any differential subring, then $L(A)=\left\{A_{p} \mid p \in \operatorname{Spec} A\right\}$ is in one-to-one correspondence with $\operatorname{Spec} A$ since $A_{P}$ $=A_{Q} \Rightarrow P=m\left(A_{P}\right) \cap A=m\left(A_{Q}\right) \cap A=Q$. We define $L_{d}(A) \subset L(A)$ to be the set of all $A_{P}$ such that $P$ is a nonzero differential prime ideal in $A$.

For the proofs of the following lemmas, we refer to [1, pp. 164-165], noting that the word "differential" may be inserted as indicated.

Lemma 1. If $A \subset K$ is a differential subring and $M$ is a local differential ring with $A \subset M<K=\mathrm{qf}(A)$, then $M$ dominates a unique member of $L_{d}(A)$.

Lemma 2. Let $M$ and $N$ be local differential subrings of $K, P=\{M, N\}=$ the differential subring generated by $M$ and $N$. Then the following are equitalent:

(1) There is a differential prime $\mathscr{L} \subset P$ such that $m(M)=\mathscr{L} \cap M, m(N)=\mathscr{L} \cap N$.

(2) There is a local differential ring $Q \geqq M, N$.

If either of these conditions holds, we say $M$ and $N$ are siblings.

LEMMA 3. If $M$ and $N$ are arbitrary differential subrings of $K$, the following are equiralent:

(1) If $A \in L(M), B \in L(N)$ are siblings, then $A=B$.

(2) $L(M) \cap L(N)=L(P)$. (P is still $\{M, N\}$.)

Let $k$ be a differential subring of $K$. We topologize $c(k)$, the set of all local differential rings in $K$ containing $k$, by taking as basic opens all sets of the form $\mathcal{C}\left(k\left\{\alpha_{1}, \ldots \alpha_{n}\right\}\right), \alpha_{i} \in K$. One checks that $\mathbb{C}\left(k\left\{\alpha_{1}, \ldots, \alpha_{m}\right\}\right) \cap \mathcal{C}\left(k\left\{\beta_{1}, \ldots, \beta_{n}\right\}\right)=$ $C\left(k\left\{\alpha_{1}, \ldots, \alpha_{m}, \beta_{1}, \ldots, \beta_{n}\right\}\right)$.

Proposition 1. If $M \in \mathbb{C}(k)$, then the topological closure $\mathrm{Cl}\{M\}$ of $\{M\}$ equals $\left\{M_{1} \in \mathbb{C}(k) \mid M \supset M_{1} \supset k\right\}$.

Proof. Let $Q$ be the set just described. If $c(k)-c(B)$ is a basic closed set containing $\{M\}$, then $M \ngtr B$ so that $M_{1} \ngtr B$ for any differential ring $M_{1} \subset M$. Thus any basic closed set containing $\{M\}$ also contains $Q$. Conversely, $M_{1} \notin M$ implies that there exists $x \in M_{1}-M$. Let $B=k\{x\}$. Then $M \in C(k)-C(B)$ and $M_{1} \notin C(k)-C(B)$, and $M_{1} \notin \mathrm{Cl}\{M\}$. 
Definition. An affine model of $K$ over $k$ is a subspace of $\mathcal{O}(k)$ of the form $L_{d}\left(k\left\{\alpha_{1}, \ldots, \alpha_{n}\right\}\right)$, where $\operatorname{qf}\left(k\left\{\alpha_{1}, \ldots, \alpha_{n}\right\}\right)=K$. A pre-model of $K$ over $k$ is a finite union of affine models. Let $M$ be a pre-model of $K$ over $k . M$ is complete if every differential place of $K$ finite on $k$ dominates some point in $M . M$ is called a model of $K$ over $k$ if $M$ has no two distinct siblings.

Several comments are in order: first of all, an affine model is indeed a model, for if $R_{\mathscr{L}_{1}} \neq R_{\mathscr{L}_{2}}$ there exists $a \in R$ such that $a$ is a unit of $R_{\mathscr{L}_{1}}$, say, but not $R_{\mathscr{L}_{2}}\left(a \in \mathscr{L}_{2}-\mathscr{L}_{1}\right)$. Then $R_{\mathscr{L}_{1}}$ and $R_{\mathscr{L}_{2}}$ cannot be siblings. Second, note that the ring of a differential place can dominate at most one member of a model. In other words, a model is "irredundant" in Zariski's terminology [4].

Let $\mathscr{M}$ be an irreducible Ritt manifold over a differential field $k$ of characteristic zero (the set of zeros in some universal extension of $k$ of a prime differential ideal $\left.P \subset k\left\{y_{1}, y_{2}, \ldots, y_{n}\right\}\right)$ with generic point $x$. Then $k\langle x\rangle$ is uniquely determined by $\mathscr{M}$ up to isomorphism over $k$, and for any $\eta \in \mathscr{M}$, the set

$$
\mathcal{O}_{\eta, \mathscr{M}}=\left\{f(x) / g(x) \mid f, g \in k\left\{y_{1}, \ldots, y_{n}\right\}, g(\eta) \neq 0\right\}
$$

is a local differential ring. Then $\left\{\mathcal{O}_{\eta, \mathscr{M}} \mid \eta \in \mathscr{M}\right\}$ is an affine model of $k\langle x\rangle$ over $k$.

Unlike its algebraic counterpart, an affine model of a differential field $K$ does not necessarily determine its coordinate ring. For example, take two nonisomorphic differentially simple rings in $K$. This shows why in some cases one needs to consider all the primes in a differential ring.

Finally, note that a maximal element of $\mathscr{C}(k)$, with respect to domination, is a ring of a differential place and conversely. Every member of $\mathcal{O}(k)$ is dominated by one of these.

Let $\phi$ be a differential place of any ordinary differential field $K$ of characteristic zero, with ring $R$ and maximal ideal $M$. The $U=R-M$ is a subgroup of $K^{*}=K$ $-\{0\}$. If $v: K^{*} \rightarrow K^{*} / U=\Gamma$ is the natural homomorphism of abelian groups where $\Gamma$ is written additively, then $\Gamma^{+}=v\left(M^{*}\right)$ is closed under addition. $\alpha=v(x) \in \Gamma^{+}$ implies $1 / x \notin R$, so that $v(1 / x)=-\alpha \notin \Gamma^{+} . x \in R$ if and only if $\alpha \in \Gamma^{+}$or $\alpha=0$. For $\alpha, \beta \in \Gamma$, we shall say that $\alpha \leqq \beta$ if $\beta-\alpha \in \Gamma^{+} \cup\{0\}=v\left(R^{*}\right)$. This relation is reflexive, antisymmetric, and transitive and hence $\Gamma$ becomes an ordered abelian group (but not necessarily totally ordered).

Let $a, x, z \in K$ and choose a differential indeterminate $y$ over $K$. The following conditions are easily verified (where $v(0)=\infty>\alpha$ for every $\alpha \in \Gamma):(1) v(x) \geqq 0$ $\Rightarrow v\left(x^{\prime}\right) \geqq 0, \quad\left(1^{\prime}\right) \quad v(x)>0 \Rightarrow v\left(x^{\prime}\right)>0 . \quad$ (2) $v(a) \leqq v(x), \quad v(z) \Rightarrow v(a) \leqq v(x+z), \quad\left(2^{\prime}\right)$ $v(a)<v(x), v(z) \Rightarrow v(a)<v(x+z)$, (3) for $a \in K$, if $v(a) \geq 0$, then there exists a differential polynomial $f \in R\{y\}$ such that $v(c)>0$ for each coefficient $c$ in $f$ and $v(f(a))=0$. (4) qf $\left(v^{-1}\left(\Gamma^{+} \cup\{\infty, 0\}\right)\right)=K$.

[Proof of (2) (resp. 2'): $x / a, y / a \in R$ (resp. $M) \Rightarrow(x+y) / a \in R$ (resp. M). (3): If $v(a) \geq 0$, then $a$ satisfies a $\phi$-special polynomial. That is, there is a polynomial $f$ such that $1=f(a)$ and the coefficients of $f$ are in $M$. Then $0=v(f(a))$.]

We call any homomorphism $v$ of $K^{*}$ into a (not necessarily totally) ordered 
abelian group $\Gamma$, satisfying the conditions (1), $\left(1^{\prime}\right),(2),\left(2^{\prime}\right),(3)$, and (4), a differential raluation of $K$.

Given a differential valuation $v$ of $K$, we can recover from it a differential place $\phi$. To see this, set $M^{*}=r^{-1}\left(\Gamma^{+}\right), U=r^{-1}(0), M=M^{*} \cup\{0\}, R=M \cup U$; then $R$ is a differential ring, $U$ is its group of units, and $M$ is a differential ideal, so that $R$ is local with maximal ideal $M$. If the natural homomorphism $\phi: R \rightarrow R / M$ were not a differential place of $K$, then it could be extended to one, say $\bar{\phi}$, and by condition (4) there would exist an element $a \in R_{\tilde{\phi}}-R$; condition (3) would imply that $a$ is a zero of a $\phi$-special differential polynomial, hence of a $\tilde{\phi}$-special one, so that $a$ could not be an element of $R_{\tilde{\phi}}$.

REMARK. Let $r$ be a differential valuation of $K$ whose ring contains $k$ and let $x_{1}, x_{2}, \ldots, x_{n} \in K^{*}$. Then there is an $x \in K^{*}$ such that $r(x) \leqq v\left(x_{1}\right), \ldots, r\left(x_{n}\right)$.

Proof. There exist nonzero elements $a_{1}, \ldots, a_{n}, b$ in the ring of $r$ such that $x_{j}=b / a_{j}$ for every $j$. Setting $z=1 /\left(a_{1} \cdots a_{n}\right)$, we see that $v(z) \leqq v\left(1 / a_{j}\right) \leqq v\left(x_{j}\right)$ for every $j$.

THEOREM 1. Let $K$ be an ordinary differential field, $k$ a differential subring of $K$ containing the rationals, with $K=\mathrm{qf}\left(k\left\{x_{1}, \ldots, x_{n}\right\}\right)$, where $x_{1}=1$. Then there exists a complete pre-model of $K$ over $k$.

Proof. Let $V$ be the set of all nontrivial differential valuations $r$ of $K$ that are finite on $k$. That is, $v \in V$ if the ring of $v$ contains $k$ and is properly contained in $K$. Then for each $v \in V$, choose an $x_{v} \in K^{*}$ such that $r\left(x_{v}\right) \leqq v\left(x_{i}\right)$, for $i=1, \ldots, n$. Set $o_{v}=k\left\{x_{1} / x_{v}, \ldots, x_{n} / x_{v}\right\}$ and $M=\bigcup_{v \in V} L_{d}\left({ }^{\prime} v\right)$. Then if $R_{v}$ is the ring of any differential place of $K$ which is finite on $k$, then $R_{v}$ dominates at least one member of $M$ (in fact, a member of $L_{d}\left(\sigma_{v}\right)$ ). We shall show that $M$ can be replaced by a finite union having the same property.

Now we can identify $V$ with $\mathscr{V}=\left\{R_{v} \mid v \in V\right\} \subset \mathcal{C}(k)$; then the induced topology on $V$ has for basic opens sets of the form

$$
B\left(k\left\{\alpha_{1}, \ldots, \alpha_{n}\right\}\right)=\left\{v \in V \mid R_{v} \supset k\left\{\alpha_{1}, \ldots, \alpha_{n}\right\}\right\} .
$$

If $S$ is a differential subring of $K$ containing $k$, let $R(S)=\mathscr{V} \cap \mathcal{O}(S)$.

Claim. There is a finite subset $F$ of $V$ such that

$$
\mathscr{V}=\bigcup_{v \in V} R\left(\|_{v}\right)=\bigcup_{v \in F} R\left(*_{v}\right)
$$

Proof. First we show that $V$ is quasi-compact (see [4, p. 113]). Any $v \in V$ is determined if for every $x \in K$, we know whether $v(x)$ is $0,+$, or $\square$ (neither one), where we take $r(0)=\infty$ to be " + ". Let $Z=\{0,+, \square\}$ so that $v$ can be considered as a map from $K$ to $Z$. Call a subset of $Z$ open if it is $\varnothing, Z$, or $\{0,+\}$ and put the product topology on $Z^{K}$. One checks that the topology on $V$ induced by that on $Z^{K}$ is identical with the topology on $V$ induced by that on $C(k)$ and so $V$ is a subspace of $Z^{K}$. As in the reference, we add the trivial differential valuation $v_{t}$ to $V$ and call the new space $V^{*}$. (The topology on $V^{*}$ is defined in the same way as on $V$.) Then $V$ 
is a subspace of $V^{*}$ and since every basic open of $V^{*}$ contains $v_{t}$, we see that $V$ is quasi-compact if and only if $V^{*}$ is.

Now introduce the discrete topology on $Z$ which is Hausdorff. Then $Z^{K}$ is compact in the new product topology by the Tychonoff Theorem. We shall show that $V^{*}$ is closed in $Z^{K}$, and hence compact, in this stronger topology, from which it follows that $V^{*}$ is quasi-compact in the weaker (original) topology.

Let $f \in Z^{K}$. Then $f \in V^{*}$ if and only if $f$ satisfies the following three conditions:

(1) $R=\{x \in K \mid f(x) \in\{0,+\}\}$ is a differential ring containing $k$.

(2) This ring is local with maximal ideal $M=\{x \mid f(x) \in\{+\}\}$ which is differential.

(3) If $f(x)=\square$, then there exists $g \in R\{y\}$, where $y$ is a differential indeterminate, such that every coefficient of $g$ is in $M$ and $g(x) \in R-M$.

These conditions are equivalent to the following three conditions:

(1') If $x, y \in K$, then either $f(x)=\square$ or $f(y)=\square$ or $f(x+y), f(x y), f\left(x^{\prime}\right)$ belong to $\{0,+\}$; and if $a \in k$, then $f(a) \in\{0,+\}$.

(2') (a) If $x, y \in K$, then either $f(x) \in\{0, \square\}$ or $f(y) \in\{0, \square\}$ or $f(x+y), f\left(x^{\prime}\right) \in\{+\}$.

(b) If $f(x) \in\{0,+\}$ and $f(y) \in\{+\}$, then $f(x y) \in\{+\}$.

(c) $f(0)=+$ (these say that $M$ is a differential ideal in $R$ ).

(d) If for $x \neq 0, f(x)=0$, then $f(1 / x) \in\{0,+\}$.

(e) $f(1)=0$ (i.e. $M$ contains precisely the nonunits in $R$ ).

$\left(3^{\prime}\right)$ Either $f(x) \in\{0,+\}$ or there exists $g \in K\{y\}$ such that $f(g(x))=0$ and $f(a)=+$ for every coefficient $a$ in $g$.

For each $x$ in $K$ the map $\pi_{x}: Z^{K} \rightarrow Z$, defined by the formula $\pi_{x}(f)=f(x)$, is continuous. Set

$$
\begin{aligned}
F_{x, y}=\left[\pi_{x}^{-1}(\square) \cup \pi_{y}^{-1}(\square) \cup \pi_{x+y}^{-1}(0,+)\right] & \cap\left[\pi_{x}^{-1}(\square) \cup \pi_{y}^{-1}(\square) \cup \pi_{x y}^{-1}(0,+)\right] \\
& \cap\left[\pi_{x}^{-1}(\square) \cup \pi_{y}^{-1}(\square) \cup \pi_{x^{1}}^{-1}(0,+)\right],
\end{aligned}
$$

a closed set. Then $\left(1^{\prime}\right)$ says that $f \in \bigcap_{x, y \in K} F_{x, y} \cap \bigcap_{x \in k} \pi_{x}^{-1}(0,+)$. (2') (a) says that $f$ is in a similar closed set, (b) says that

$$
f \in \bigcap_{x, y \in K}\left[\pi_{x}^{-1}(\square) \cup \pi_{y}^{-1}(0, \square) \cup \pi_{x y}^{-1}(+)\right],
$$

(c) says that $f \in \pi_{0}^{-1}(+)$, (d) says that $f \in \bigcap_{x \in K^{*}} G_{x}$, where $G_{x}=\pi_{x}^{-1}(\square,+) \cup$ $\pi_{1 / x}^{-1}(0,+)$, and (e) says that $f \in \pi_{1}^{-1}(0)$. Finally, $\left(3^{\prime}\right)$ asserts that

$$
f \in \bigcap_{x \in K ; g(x) \in K(x)}\left[\pi_{x}^{-1}(0,+) \cup \pi_{g(x)}^{-1}(0)\right] \cap\left[\pi_{x}^{-1}(0,+) \cup \bigcap_{m=\text { coeff in } g} \pi_{m}^{-1}(+)\right] .
$$

Since these are all closed sets, $V^{*}$ is closed and, as remarked earlier, $V$ is quasicompact. This proves the claim.

To complete the proof of the theorem, note that $R\left(o_{v}\right)$ is open in $\mathscr{V}$ since $R\left(a_{v}\right)$ $=\mathscr{V} \cap \mathcal{O}\left(o_{v}\right)$, and $\bigcup_{v \in V} R\left(o_{v}\right)$ is an open cover of $\mathscr{V}$ because given any $v \in V$, $R_{v} \in R\left(o_{v}\right)$. Thus there is a finite subcover $\bigcup_{v \in F} R\left(o_{v}\right)$. Let $M_{1}=\bigcup_{v \in F} L_{d}\left(o_{v}\right)$. Then $M_{1}$ is the required pre-model since if $R_{v}$ is a differential valuation ring containing $k$, then $R_{v} \in R\left(o_{w}\right)$ for some $w \in F$, and hence $R_{v}$ dominates some member of $L_{d}\left(o_{w}\right)$. Q.E.D. 
Before proceeding to the question of the existence of complete models of a differential field, we need to derive more information concerning the structure of differential valuation rings.

Let $k$ be an ordinary differential field of characteristic zero. Kolchin has shown that if $P \in k[y]$ is a cubic polynomial with distinct roots and if $\mathscr{L}$ denotes the general component of the differential polynomial $A=z y^{\prime 2}-P \in k\{y, z\}$ having $(\eta . \zeta)$ as generic zero, then there is a differential homomorphism $\phi: k\{\zeta\} \rightarrow k$ with $\zeta \mapsto 0$ and no element $\alpha$ in any extension of $k$ such that $\phi$ extends to a differential homomorphism $k\{\eta, z\} \rightarrow k\{\alpha\}$ or to a differential homomorphism $k\left\{\eta^{-1}, \zeta\right\} \rightarrow k\{\alpha\}$.

Now let $K$ be an ordinary differential field of characteristic zero, $R$ a differential valuation ring with quotient field $K$. Then for certain $x \in K-R$ we can prove that a different expression in $x$, not its reciprocal in general, will belong to the maximal ideal of $R$.

Definition. Let $\phi$ be a differential place of the differential field $K$, and let $x \in K$. We say that $x$ is $\phi$-reducible if there exist a polynomial $1+f \in R_{\phi}\{y\}$ and a natural number $i$ such that $y^{(i)}$ divides $f$ and $1+f(x)=0$.

Proposition 3. Let $v$ be a differential valuation of $K$ with ring $R$ and let $\phi$ be the corresponding differential place. Let $x$ be $\phi$-reducible, $x \notin R$. Then there exist nonnegative integers $n, N, i_{0}, \ldots, i_{n}$, with at least one $i_{j}>0$ such that $1 / z^{N} x^{i_{o}} \cdots\left(x^{(n)}\right)^{i_{n}} \in M$ (the maximal ideal in $R$ ) where $z$ is any element of $K$ such that $v(z) \leqq 0, v(x), \ldots, v\left(x^{(n)}\right)$.

Notice that if we allow all the $i_{j}$ to be zero, the proposition is trivially true since $v(z) \neq 0$.

Proof. There exist a differential polynomial $f=\sum_{j=1}^{p} m_{j} y^{i_{j}} \cdots\left(y^{(n)}\right)^{i_{n}}$ with $m_{1}, \ldots, m_{p} \in R$, and an integer $l$ with $0 \leqq l \leqq n$, such that $i_{1_{l}}, \ldots, i_{p_{l}}>0$ and $1+f(x)$ $=0$. Choose $z \in K$ such that $v(z) \leqq 0, v(x), \ldots, v\left(x^{(n)}\right)$; for each index $k$ set $i_{k}=$ $\min \left\{i_{1_{k}}, \ldots, i_{p_{k}}\right\}$, and let $N=1+\max _{1 \leqq j \leqq p}\left\{i_{j_{0}}+\cdots+i_{j_{n}}\right\}$, and set $r=z^{N} x^{i_{0}} \cdots\left(x^{(n)}\right)^{i_{n}}$. Then $i_{l}>0$. Also

$$
\left[x^{i_{j}} \cdots\left(x^{(n)}\right)^{i_{j}}\right] / r=\left[(x / z)^{i_{j_{0}}-i_{0}} \cdots\left(x^{(n)} / z\right)^{i_{j_{n}}-i_{n}}\right] \cdot\left[z^{-N+\sum_{k=0}^{n}\left(i_{j_{k}}-i_{k}\right)}\right]
$$

which is in $M$ so that $v\left(x^{i_{j}} \cdots\left(x^{(n)}\right)^{i_{j_{n}} / r}\right)>0$ for every $j$, whence

$$
v(1 / r)=v\left(-\sum_{j=1}^{p} m_{j} x^{i_{j 0}} \cdots\left(x^{(n)}\right)^{i_{j n} / r}\right)>0 \quad \text { and } \quad 1 / r \in M . \quad \text { Q.E.D. }
$$

Let $A$ be an arbitrary integral domain. One sees easily that $A$ is a valuation ring if and only if qf $(A)-A$ is multiplicatively closed. Half of this result can be generalized to differential algebra, namely

THEOREM 2. If $R$ is the ring of a differential place $\phi$ of an ordinary differential field $K$ of characteristic zero and $s, \bar{s} \in K-R$, then there exist nonnegative integers $i, j$ such that $s^{(i)} \bar{s}^{(j)} \notin R$. 
Proof. Suppose that for all $i, j \geqq 0, s^{(i)} \bar{s}^{(j)} \in R$. There is a unit $b$ in $R$ such that $b=p_{0}+\sum_{j=1}^{n} p_{j} s^{i_{j 0}} \cdots\left(s^{(r)}\right)^{i_{j_{r}}}$, where the $p_{i}$ are nonunits in $R$. We may assume $p_{0}=0$ by replacing $b$ by $b-p_{0}$ if necessary.

We assume $[1]=\left(i_{1_{r}}, \ldots, i_{1_{r}}\right), \ldots,[n]=\left(i_{n_{0}}, \ldots, i_{n_{r}}\right)$ are in lexicographic increasing order with respect to $N([i])=\sum_{k=0}^{r} i_{j_{k}}, i_{j_{0}}, \ldots, i_{j_{r}}$ and that $b$ is chosen so that $N([n])$ is minimal. Let $M([n])=N([n]) \cdot$ (the number of times $N([n])$ is assumed in b). Similarly

$$
\bar{b}=\sum_{j=1}^{m} \bar{p}_{j} \bar{s}^{i_{j}} \cdots\left(\bar{s}^{(r)}\right)^{i_{j r}}
$$

where $N([m])$ is minimal.

Suppose $N([n]) \geqq N([m])$ (we know $N([m])>0$ ). Then

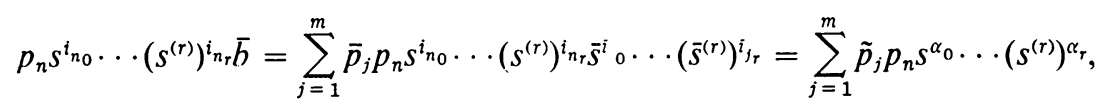

where the $\tilde{p}_{0}, \ldots, \tilde{p}_{m}$ are nonunits in $R$ and $0 \leqq N([\alpha])<N([n])$. Hence

$$
b \bar{b}=\sum_{j=1}^{n-1} \bar{b} p, s^{i_{j 0}} \cdots\left(s^{(r)}\right)^{i_{j_{r}}}+\sum_{k=1}^{m} \tilde{p}_{k} p_{n} s^{\alpha_{0}} \cdots\left(s^{(r)}\right)^{\alpha_{r}} .
$$

But $b \bar{b}$ is a unit in $R$ so that if $M([n])=N([n])$ this contradicts the minimality of $b$. If $M([n]) / N([n])=t>1$, then repeating the argument $t$ times contradicts the minimality of $b$. Q.E.D.

COROLlaRY 1. If $x$ is a constant in $K$, then $x$ or $1 / x$ is in $R$.

COROLlaRY 2. If $x$ is a constant in $K, n$ a positive integer such that $x^{n} \in R$, then $x \in R$. Thus if $v$ is the corresponding differential valuation, then $n v(x) \geqq 0$ implies $v(x) \geqq 0$.

Now we are ready to write down a certain condition which if satisfied by a differential field implies the existence of a complete model of that field.

As before, let $k$ be a differential ring containing the rationals and contained in a differential field $K$. Then if $a, b \in K$, we write $a \equiv b(\bmod k)$ if $a / b$ is a unit in $k$. We shall omit the " $\bmod k$ " when no confusion can arise. This is clearly an equivalence relation and now we can state

THEOREM 3. Let $k, K$ be as above with $K=\mathrm{qf}\left(k\left\{x_{1}, \ldots, x_{n}\right\}\right)$, where the $x_{i}$ are not necessarily differential indeterminates, satisfying the following condition: $(+)$ if $v$ is a differential valuation of $K$ whose ring $R_{v}$ contains $k$, then $x_{i}$ can be written in the form $x_{i} \equiv f_{i v} / g_{i v}, i=1,2, \ldots, n$, where $f_{i v}, g_{i v} \in R_{v}$ and such that whenever $w$ is any other differential valuation of $K$ whose ring contains $k$, we have

$$
g_{i w} / g_{i v} \in k\left\{f_{1 w}, \ldots, f_{n w}, g_{1 w}, \ldots, g_{n w}\right\}=o_{w} .
$$

Then there exists a complete model of $K$ over $k$. 
Proof. Let $V$ be the set of all differential valuations $v$ of $K$ such that $R_{v} \supset k$. For each $v \in V$, write $x_{i} \equiv f_{i v} / g_{i v}, i=1, \ldots, n$, and form $o_{v}$ as in (+). Then the quotient field of $\rho_{v}$ is $K$ and $R_{v} \supset \rho_{v}$. By the proof of Theorem 1, there is a finite subset $F$ of $V$ and a complete pre-model $M=\bigcup_{v \in F} L_{d}\left(o_{v}\right)$. We claim $M$ is in fact a model.

Suppose there exists $u \in V$ and $v, w$ in $F$ such that $R_{u} \geqq\left(\sigma_{v}\right)_{P_{v}}$ and $R_{u} \geqq\left(\sigma_{w}\right)_{P_{w}}$ for some differential prime ideals $P_{v}, P_{w}$ in ${ }^{\prime} v$, "w respectively. Set $\mathcal{O}_{v}=\left(o_{v}\right)_{P_{v}}$ and $\mathcal{O}_{w}=\left(o_{w}\right)_{P_{w}}$. Then there is a differential prime ideal $\mathscr{L} \subset_{o}=\left\{o_{v}, o_{w}\right\}$ such that $\sigma_{L} \geqq \mathcal{O}_{v}, \mathcal{O}_{w}$. We shall show ${ }^{\prime} \mathscr{L}=\mathcal{O}_{v}$. It suffices to show $n \subset \mathcal{O}_{v}$ for in that case if $\alpha / \beta$

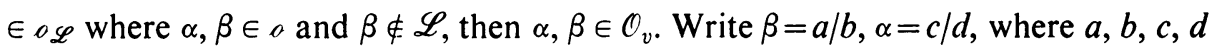
$\in o_{v}, b, d \notin P_{v}$, so that $\alpha / \beta=c b / d a$ with $d a \notin P_{v}$ (if $a \in P_{v}$, then $b \beta=a$ implies $\beta \in \mathscr{L}$ ). Hence $\alpha / \beta \in \mathcal{O}_{v}$ and $\sigma_{\mathscr{L}} \subset \mathcal{O}_{v}$. Thus we are reduced to showing that ${ }_{0}{ }_{w} \subset \mathcal{O}_{v}$, i.e. that $f_{i w}, g_{i w} \in \mathcal{O}_{v}$ for $i=1, \ldots, n$. Now $f_{i w} \equiv g_{i w} x_{i} \equiv g_{i w} f_{i v} g_{i v}^{-1}=f_{i v} /\left(g_{i v} / g_{i w}\right)$ and $g_{i w}=$ $\left(g_{i w} / g_{i v}\right) g_{i v} \equiv g_{i v} /\left(g_{i v} / g_{i w}\right)$ for all $i=1,2, \ldots, n$. By $(+), g_{i v} / g_{i w} \in o_{v}$, and $g_{i w} / g_{i v} \in o_{u}$ so that $u\left(g_{i v}\right) \geqq u\left(g_{i w}\right)$ and $u\left(g_{i w}\right) \geqq u\left(g_{i v}\right)$. Hence $u\left(g_{i v}\right)=u\left(g_{i w}\right)$, so that $g_{i v} / g_{i v} \notin P_{v}$, and thus $f_{i w}, g_{i w} \in \mathcal{O}_{v}$. It follows that $\mathscr{L}_{\mathscr{L}} \subset \mathcal{O}_{v}$. Similarly $\sigma \mathscr{L}=\mathcal{O}_{w}$. Q.E.D.

EXAMPLE. Let $k$ be an ordinary differential field of characteristic zero and let $x$ be an exponential of an integral of any element of $k$, so that $x^{\prime} / x=d \in k$. We claim there is a complete model of $k\langle x\rangle$ over $k$.

Let $v$ be a differential valuation of $k\langle x\rangle$ whose ring $R$ contains $k$. We first show that $x$ or $1 / x$ belongs to $R$.

Proof. If not, then by Theorem 2 there exist nonnegative integers $i, j$ such that $x^{(i)}(1 / x)^{(j)} \notin R$. But $x^{(i)}=d_{1} x$ and $(1 / x)^{(j)}=d_{2} / x$, where $d_{1}, d_{2} \in k$. This is a contradiction.

To complete the proof, we shall show that $k\langle x\rangle$ satisfies the condition (+).

Let $V$ be the set of differential valuations $v$ of $k\langle x\rangle$, whose ring $R_{v}$ contains $k$; for each $v \in V$, write $x=x / 1$ if $x \in R_{v}$ (so that $f_{v}=x$ and $g_{v}=1$ ) and write $x=1 /(1 / x)$ if $x \notin R_{v}$. Then if $v, w \in V$, one sees by checking cases that $g_{w} / g_{v} \in k\left\{f_{w}, g_{w}\right\}$. Thus there exists a complete model of $k\langle x\rangle$ over $k$.

Moreover, we claim that if $v \in V$ and $v(x) \neq 0$, then the value group $\Gamma_{v}$ of $v$ is isomorphic to $Z$; indeed if $f(x) \in k\{x\}$ has degree $n$ and $v(x)>0$, then we can write $f(x) / x^{n}=m_{1}(x) / x^{n}+\cdots+m_{k}(x) / x^{n}$ where the $m_{i}(x)$ are homogeneous differential polynomials of decreasing degrees, and this equals $c_{0}+c_{1} / x^{l_{1}}+\cdots+c_{k} / x^{l_{k}}$, where $v\left(c_{i}\right)=0$ for all $i$ and $l_{1}<\cdots<l_{k}=n-l(l$ denotes the lowest degree of any monomial appearing in $f$ ).

Now, in general, if $v\left(x_{1}\right)<v\left(x_{2}\right), \ldots, v\left(x_{n}\right)$, then $v\left(\sum_{i=1}^{n} x_{i}\right)=v\left(x_{1}\right)$; in fact, $\sum_{i=1}^{n} x_{i} / x_{1}=1+x_{2} / x_{1}+\cdots+x_{n} / x_{1} \in R_{v}-M_{v}$.

Hence in our example, $v(f(x))=n v(x)-(n-l) v(x)=l v(x)$. Therefore, if $f(x) / g(x)$ represents any element of $R_{v}, v\left(f(x) / g(x)\right.$ ) equals $l_{f}-l_{g}$ where $l_{f}$ (resp. $l_{g}$ ) denotes the lowest degree of any monomial appearing in $f$ (resp. $g$ ).

On the other hand, if $v(x)<0$, we merely get the integers with the reverse order by considering $1 / x$. Thus $\Gamma_{v}$ is totally ordered. Note that if $v(x)=0$, the argument breaks down. 
We now consider the question of higher dimensional examples, beginning with the following generalization of Corollary 1 to Theorem 2 .

Proposition. Let $K$ be a differential field, $z \in K$ and $v$ a differential valuation of $K$ with ring $R$ such that $z^{\prime} / z \in R$. Then either $z$ or $1 / z$ belongs to $R$.

Proof. If not, there exist nonnegative integers $i, j$ not both zero such that $z^{(i)}(1 / z)^{(j)} \notin R$. Let $n=i+j>0$. But $(1 / z)^{(j)}=f_{j}(z) / z^{j+1}$ or zero, where $f_{j}$ is a homogeneous differential polynomial in $z$ of degree $j$ with integral coefficients (this is easily seen by induction). Then $z^{(i)}(1 / z)^{(j)}=\bar{f}_{j+1}(z) / z^{j+1} \notin R$ where $\bar{f}_{j+1}$ is homogeneous of degree $j+1$, and hence some monomial $m_{j+1}(z) / z^{j+1} \notin R, m_{j+1}$ also being of degree $j+1$. But $z^{\prime} \mid z \in R$ implies $z^{(n)} / z \in R$ for all $n=0,1, \ldots$ (again by induction), so that $m_{j+1}(z) / z^{j+1} \in R$, a contradiction. Q.E.D.

COROLlaRY 1. Let $k$ be a subfield of $K$ (ordinary differential fields of characteristic zero, as usual) such that $K=k\left\langle x_{1}, \ldots, x_{n}\right\rangle$.

Then there exists a complete model of $K$ over $R$, where $R=k\left\{x_{1}^{\prime} / x_{1}, \ldots, x_{n}^{\prime} / x_{n}\right\}$.

Proof. If $v$ is a differential valuation of $K$ whose ring contains $R$, set $f_{i v}=x_{i}$, $g_{i v}=1$ if $x_{i}$ belongs to the ring of $v$ and $f_{i v}=1, g_{i v}=1 / x_{i}$ if $x_{i}$ does not belong to the ring of $v$. Then check condition $(+)$.

COROllary 2. If $\phi: S \rightarrow U$ is a differential homomorphism of a differential domain $S$ into a differential field $U$, both of characteristic zero, and if $z \in \operatorname{qf}(S)$ is such that $\phi$ does not extend to $z$ or $1 / z$, then $\phi$ does not extend to $z^{\prime} / z$.

COROLlaRY 3. Let $v$ be a differential valuation of $K$ with differential value group $\Gamma_{v}$. Then $\Gamma_{v}$ is torsion free.

Proof. If $n$ is a nonzero integer such that $n v(x)=0$ for some $x \in K$, then $x^{n}$ is a unit in $R$, the differential valuation ring of $v$. Hence $x^{n-1} x^{\prime} / x^{n}=x^{\prime} / x \in R$. Thus $v(x)$ must compare with zero. If $v(x)$ is strictly positive or negative, the same is true of $n v(x)$. Hence $v(x)=0$.

As a final example, let $k$ be an ordinary differential field of characteristic zero, $y$ a differential indeterminate over $k, d \in k^{*}$. Let $A=y^{\prime \prime} y-y^{\prime 2}-d y y^{\prime} \in k\{y\}$; let $P_{g}$ be the general component of $\{A\}$ in $k\{y\}$ and let $x$ be the residue of $y$ modulo $P_{g}$. We shall prove the existence of a complete model of $K=k\langle x\rangle$ over $k$. (Remark. For a concrete example, take $C\left\langle e^{e^{x}}\right\rangle$-in that case $d=1$.) Note that $k\langle x\rangle=k\left(x, x^{\prime}\right)$, a pure transcendental extension of $k$ of algebraic dimension two.

Now $\left(x^{\prime} / x\right)^{\prime} /\left(x^{\prime} / x\right)=\left(x x^{\prime \prime}-x^{\prime 2} / x^{2}\right) \cdot\left(x / x^{\prime}\right)=d \in k^{*}$ so that if $R_{v}$ is any differential valuation ring of a differential valuation $v$ of $K$ over $k$, we have $x^{\prime} / x \in R_{v}$ or $x / x^{\prime}$ $\in M_{v}$, the maximal ideal in $R_{v}$. It suffices to show that $x$ or $1 / x$ belongs to $R_{v}$ for in that case one can show that condition $(+)$ is satisfied by $K$.

If not, then $x / x^{\prime} \in M_{v}$, i.e. $v(x)>v\left(x^{\prime}\right)$. Let $\delta=v(x)-v\left(x^{\prime}\right)$, a positive element of $\Gamma_{v}$, the differential value group. Also set $v_{i}=v\left(x^{(i)}\right), i=0,1, \ldots$ We claim that $v_{i}=v_{i+1}+\delta$, or equivalently, $x^{(i)} / x^{(i+1)}=\left(x / x^{\prime}\right) u$, where $u$ is a unit in $R_{v}$. 
We first establish by induction that $v_{\imath}>v_{i+1}$ for all $i$. The case $i=0$ is clear. Suppose $x^{(i)}=x^{(i+1)} \bar{m}$, where $\bar{m} \in M_{v}$. Then $x^{(i+1)}\left(1-\bar{m}^{\prime}\right)=x^{(i+2)} \bar{m}$, where the left side has value $v_{i+1}$ and the right side has value strictly greater than $v_{i+2}$. Thus $v_{0}>v_{1}>\cdots>v_{i}>\cdots$.

The claim is certainly true for $i=0$. Suppose $u x^{(i)}=x^{(i+1)} m$, where $u$ is a unit in $R_{v}$ and $m=x / x^{\prime}$ so that $v(m)=\delta$. Then $u^{\prime} x^{(i)}+u x^{(i+1)}=x^{(i+1)} m^{\prime}+x^{(i+2)} m$ $=\left(-d x^{(i+1)}+x^{(i+2)}\right) m$. The value of the right side is $v_{i+2}+\delta$ and that of the left side is $v_{i+1}$ since $v\left(u^{\prime} x^{(i)}\right) \geqq v_{i}$. This proves the claim.

Next we shall show that if $a$ and $b$ are integers, $a \neq 0$, with $a v_{0}+b \delta \geqq 0$ in $\Gamma_{v}$, then $a v_{0}+(b-1) \delta \geqq 0$. In fact, suppose $x^{a}\left(x / x^{\prime}\right)^{b} \in R_{v}$. Then $a x^{a-1} x^{\prime}\left(x / x^{\prime}\right)^{b}+b x^{a}\left(x / x^{\prime}\right)^{b-1}$ $\cdot\left(-d x / x^{\prime}\right) \in R_{v}$. The second summand has value $a v_{0}+b \delta$ and hence belongs to $R_{v}$. Thus the same is true of the first summand and we see that $a v_{0}+(b-1) \delta \geqq 0$.

In particular, $v_{0}$ and $\delta$ are rationally independent for if $a v_{0}+b \delta=0, a \neq 0$, then $a v_{0}+(b-1) \delta \geqq 0$. But this is impossible since the latter value is strictly smaller than the former. Hence $a=b=0$.

Let $z=1 / x$. Then $k\langle z\rangle=K\langle x\rangle$ and in fact $z z^{\prime \prime}-z^{\prime 2}=d z z^{\prime}, v(z)=-v_{0}$ does not compare with zero, $z / z^{\prime}=-x / x^{\prime}, v\left(z^{(i)}\right)=v\left(z^{(i+1)}\right)+\delta$ exactly as before.

Now since $x^{(i)}=m_{i} x^{(i+1)}, m_{i} \in M_{v}, i=0,1,2, \ldots$, we have the following chain of rings: $R_{v}[x] \subset R_{v}\left[x^{\prime}\right] \subset \ldots \subset R_{v}\left[x^{(i)}\right] \subset \ldots$ with $R_{v}\{x\}=\bigcup_{i=0}^{\infty} R_{v}\left[x^{(i)}\right]$. We must have $1 \in M_{v} \cdot R_{v}\{x\}$ since $R_{v}$ is a differential valuation ring and $x \notin R_{v}$. Thus $1 \in M_{v}$ - $R_{v}\left[x^{(i)_{0}}\right]$ for some $i_{0}$. Therefore $\left[x^{\left(i_{0}\right)}\right]^{-1}$ belongs to every proper prime ideal in the integral closure of $R_{v}$ in its quotient field (see [4, p. 325]). Thus if $R_{\bar{v}}$ is a valuation ring containing $R_{v},\left[x^{\left(i_{0}\right)}\right]^{-1}$ belongs to the maximal ideal of $R_{\bar{v}}$.

Let $s=x^{\left(i_{0}\right)}$ so that $s^{-1}$ is integral over $R_{v}$, and hence $s^{-1}$ is quasi-integral over $R_{v}$, i.e. there is a $d_{1} \in R_{v}, d_{1} \neq 0$, such that $d_{1}\left(s^{-1}\right)^{r} \in R_{v}$ for all $r=0,1, \ldots$ By a theorem of Seidenberg, [3, p. 168], $\left(s^{-1}\right)^{\prime}$ is also quasi-integral over $R_{v}$. Thus there is a $d_{2} \in R_{v}$ such that $\left(x^{\left(i_{0}+1\right)} / x^{\left(i_{0}\right)^{2}}\right)^{r} d_{2} \in R_{v}, r=0,1, \ldots$ Applying $v$, we see that for $r=0,1, \ldots, r\left(v_{i_{0}+1}-2 v_{i_{0}}\right)+\gamma \geqq 0$, where $\gamma \geqq 0$. Then $r\left(v_{0}-\left(i_{0}+1\right) \delta-2 v_{0}+2 i_{0} \delta\right)$ $+\gamma \geqq 0$, or $r\left(-v_{0}+\left(i_{0}-1\right) \delta\right)+\gamma \geqq 0, r=0,1, \ldots$ Therefore $-r v_{i_{0}-1}+\gamma \geqq 0$ for all $r$ and thus $d_{2} /\left(x^{\left(i_{0}-1\right)}\right)^{r} \in R_{v}$. But this means that $1 / x^{\left(i_{0}-1\right)}$ is quasi-integral over $R_{v}$. Continuing, we see that $1 / x$ is quasi-integral over $R_{v}$.

LEMMA. Let $R$ be a domain with $\mathrm{qf}(R)=K, z \in K$, such that $z$ is quasi-integral over $R$. Then $z$ belongs to the intersection of all rank one valuation rings of $K$ which contain $R$.

Proof. Suppose $d_{1} z^{r} \in R$ for $r=0,1, \ldots$ where $d_{1} \in R$, and let $\tilde{v}$ be a rank one valuation whose ring contains $R$. Then $\tilde{v}\left(d_{1}\right)+r \tilde{v}(z) \geqq 0$ for all $r$, so that $\tilde{v}\left(d_{1}\right)$ $\geqq-r \tilde{v}(z), r=0,1, \ldots$ If $\tilde{v}(z)<0$, then $-\tilde{v}(z)>0$, and by the Archimedean property there is an $r$ such that $r(-\tilde{v}(z))>\tilde{v}\left(d_{1}\right)$, a contradiction.

Thus, in our example, $z=1 / x$ belongs to all rank one valuation rings which contain $R_{v}$ and the same is true for $1 / s,(1 / s)^{\prime},(1 / s)^{\prime \prime}$, etc. Suppose we could find a rank one place $\psi$ of $K$ over $k$ whose ring dominates $R_{v}$. Then since $1 / s \notin R_{v}$, we know 
that $1 / s$ satisfies a $\phi$-special differential polynomial, where $\phi$ is the differential place corresponding to $r ;(*) \sum b_{1}(1 / s)^{[i]}=1, b_{i} \in M_{r}$. Then, applying $\psi$ we get the contradiction $1=0$.

Thus if $\phi$ is any place extending $\phi$ we must have rank $\phi=2$, and hence the dimension of $\phi$ is zero. Let $R_{\bar{v}}$ be the corresponding valuation ring with nonzero prime ideals $\mathscr{L}_{1}<\mathscr{L}_{2}$. Then $\left(R_{\bar{v}}\right)_{\mathscr{L}_{1}}$ is a discrete, one-dimensional, rank one valuation ring containing $R_{\bar{v}}$, call it $R_{\tilde{v}}$, with valuation $\tilde{v}$.

We shall show $\tilde{v}\left(z^{(i)}\right)>0$ for all $i$ so that we obtain the contradiction $1=0$ by applying $\tilde{\phi}$ to the equation $\sum b_{t} z^{[i]}=1$ analogous to $(*)$. Now $z / z^{\prime}=-m$ in our previous notation, and $\tilde{v}(m) \geqq 0$. We saw that $r\left(z^{(i)}\right)=v\left(z^{(i+1)}\right)+\delta$. Then for $i=0,1, \ldots$, $z^{(i)} u_{1}=z^{(i+1)} m$, where $u_{i}$ is a unit in $R_{\tilde{v}}$. If $\tilde{v}(m)>0$, then $\tilde{v}(z)>\tilde{v}\left(z^{\prime}\right)>\cdots>\tilde{v}\left(z^{(i)}\right)$ $>\cdots$ would be a strictly decreasing sequence of nonnegative integers since each $z^{(i)}$ is quasi-integral over $R_{t}$. Therefore $\tilde{v}(m)=0$. But in that case, $\tilde{v}\left(z^{(i)}\right)=\tilde{v}(z)$ for all $i$. If $\tilde{u}(z)=0$, then since $x^{(i)} u_{i}=x^{(i+1)} m$, where $u_{i}$ is a unit in $R_{\tilde{r}}$, we would have that $\tilde{v}\left(x^{(i)}\right)=\tilde{v}(x)=0$ for all $i$. But we saw that $\tilde{v}\left(x^{\left(i_{0}\right)}\right)<0$. It follows that there is no such $v$ and therefore there exists a complete model of $k\langle x ;$ over $k$.

Let $M$ and $M^{\prime}$ be models of a differential field $K$. Then if there is a differential valuation ring $R$ which dominates $\mathbb{C}_{1} \in M$ and $\mathbb{C}_{2} \in M^{\prime}$, we say $\mathbb{C}_{1}$ corresponds differentially to $\mathbb{C}_{2}$. If $M^{\prime}$ is complete, then for every $\mathscr{C}_{1} \in M$ there is an $\mathcal{O}_{2} \in M^{\prime}$ such that $\mathbb{U}_{1}$ corresponds differentially to $\mathbb{U}_{2}$.

REMARK. If $\mathscr{C}_{1}$ and $\mathscr{C}_{2}$ are differentially corresponding points on models of a differential field $K$ with $\mathscr{O}_{1} \subset \mathscr{C}_{2}$ then $\mathscr{U}_{1} \leqq \mathcal{O}_{2}$, for if $R$ is a differential valuation ring dominating both $\mathbb{C}_{1}$ and $\mathbb{C}_{2}$ and if $x$ is a nonunit in $\mathcal{O}_{1}$, then $x \in m(R)$ implies $x \in m\left(\mathbb{O}_{2}\right)$. Moreover, if $M$ and $M^{\prime}$ are models of $K$ and $\mathbb{U}^{\prime} \in M, \mathbb{C}^{\prime} \in M^{\prime}$ are such that $\mathbb{C}^{\prime} \leqq \mathbb{C}$, then $\mathbb{C}^{\prime}$ is the unique point of $M^{\prime}$ corresponding differentially to $\mathcal{O}$; indeed, if $R$ is any differential valuation ring of $K$ which dominates $\mathcal{C}^{\prime}$, then $R \geqq \mathcal{O}^{\prime}$, and if for some $\mathcal{C}^{\prime \prime} \in M^{\prime}, R \geqq \mathcal{C}^{\prime \prime}$, then $\mathcal{O}^{\prime}=\mathcal{O}^{\prime \prime}$ because $M^{\prime}$ is a model. Under certain conditions we can assert the converse.

Proposition. Let $k$ be an ordinary differential field of characteristic zero, $K a$ differential extension field of $k$. Suppose $M$ and $M_{1}$ are models of $K$ over $k$ with $M_{1}$ complete over $k$ and that $\mathcal{O} \in M$ is differentially integrally closed and such that there is no differential prime ideal $Q$ with $0<Q<m(\mathcal{O})$. Then if $\mathcal{O}$ corresponds differentially to a unique element $\mathcal{O}_{1} \in M_{1}$, then $\mathcal{O}_{1} \leqq \mathcal{O}$.

Proof. It suffices to show $\mathcal{C}_{1} \subset \mathcal{C}$. Now $C^{\prime}=\bigcap_{R_{v}} \mathcal{C} R_{v}$ where $R_{v}$ is a ring of a nontrivial differential place of $K$ over $k$. We claim $\mathcal{O}=\bigcap_{R_{v} \geqq \mathbb{C}} R_{v}$. In fact, if $R_{v} \supset \mathcal{C}=o \mathscr{L}$, then $M_{v} \cap \mathscr{C} \subset m(\mathcal{O})=\mathscr{L}_{"} \mathscr{L}$ so that $M_{v} \cap " \subset \mathscr{L}$. But $M_{v} \cap o$ is a nonzero differential prime ideal since qf $(\omega)=K$ and $v$ is nontrivial. Thus $M_{v} \cap 0=\mathscr{L}$ and therefore $R_{\iota} \geqq \mathcal{C}$ and the claim is established.

Now if $R$ is a differential valuation ring such that $R \geqq \mathbb{C}$, then $R \geqq \mathbb{C}_{1}$, since $M_{1}$ is complete and $\mathscr{C}_{1}$ is the only member of $M_{1}$ corresponding differentially to $\mathbb{C}$. Thus $\mathbb{U}_{1} \subset \mathcal{O}$. Q.E.D. 


\section{REFERENCES}

1. A. Grothendieck, Éléments de géometrie algébrique. I, Publ. Math. Inst. Hautes Études Sci. IHES, Paris, 1960.

2. J. F. Ritt, Differential algebra, Amer. Math. Soc. Colloq. Publ., Vol. 33, Amer. Math. Soc., Providence, R. I., 1950.

3. A. Seidenberg, Derivations and integral closure, Pacific J. Math. 16 (1966), 167-173.

4. O. Zariski and P. Samuel, Commutative algebra. II, Van Nostrand, Princeton, N. J., 1960.

\section{UNIVERSITY OF CALIFORNIA,}

Berkeley, California

COLUMBia University,

New YoRK, New YorK 